\title{
Reconfiguration of Solar Panels: Mathematical Model and Analysis
}

\author{
Thanh Ngo Ngoc ${ }^{1}$, Ninh Nguyen Quang ${ }^{2, *}$, Linh Bui Duy ${ }^{3}$ \\ ${ }^{1}$ Faculty of Information Technology, Electric Power University, Hanoi, Vietnam \\ ${ }^{2}$ Center for Research and Development on Renewable Energy Technology, Institute of Energy Science, IES-VAST, Hanoi, Vietnam \\ ${ }^{3}$ National Load Dispatch Centre, Vietnam Electricity, Hanoi, Vietnam
}

Email address:

nqninh@ies.vast.vn (N. N. Quang)

${ }^{*}$ Corresponding author

\section{To cite this article:}

Thanh Ngo Ngoc, Ninh Nguyen Quang, Linh Bui Duy. Reconfiguration of Solar Panels: Mathematical Model and Analysis. American Journal of Electrical Power and Energy Systems. Vol. 8, No. 5, 2019, pp. 104-110. doi: 10.11648/j.epes.20190805.11

Received: August 2, 2019; Accepted: September 2, 2019; Published: September 17, 2019

\begin{abstract}
Balance solar radiation between photovoltaic panels or between groups of photovoltaic panels will help to improve the efficiency of electricity generation of the entire solar power system, making the most of the solar energy converted into electricity power. In order to achieve this, it is necessary to reconfigure the connection of photovoltaic panels or groups of photovoltaic panels in the solar power system. In this paper, the general mathematical model for implementing resconfiguring of photovoltaic panels or groups of photovoltaic panels is presented in 2 parts: calculating to find out the optimal configuration connecting photovoltaic panels or groups of photovoltaic panels to achieve the highest performance of the solar system; and find out the best way to switch from the initial connection configuration state to the optimal configuration. The author focuses on analyzing and proposing mathematical models for two main problems in previous studies, developing objective functions and clear constraints, which are the rationale for evaluation of the quality and accuracy of the proposed algorithms, thereby developing more optimal algorithms than previous algorithms. Several experimental studies have been applied on a system of 4 solar panels. The archived results demonstrated the correctness and efficiency of the proposed mathematical model and optimal algorithm.
\end{abstract}

Keywords: Optimal, Reconfiguration, Mathematical Model, Solar Power System

\section{Introduction}

Capacity generated by Photovoltaic panels in actual condition is often lower than standard working conditions [16]. The main reasons for reducing the performance of Photovoltaic panels are solar radiation, temperature or the aging of photovoltaic panels [7-10].

The effects of solar radiation on the working process of the solar energy system (solar energy) and restructuring strategy to increase the working efficiency for the solar energy system in inhomogeneous radiation conditions has been researched and published at [11-14]. In essence, system restructuring is to change the connection of photovoltaic panels to achieve the optimal connection configuration, giving the system the maximum capacity. Currently, the restructure problem is applied to 2 main connection models of photovoltaic panels,
Series-Parallel (SP) and TCT. In [11-14], the author has proposed a method to improve the performance of the solar energy system for TCT connection circuit.

The current grid based solar energy system include the basic components such as: photovoltaic panels, energy converter, charged, load and grid. When photovoltaic panels receive solar radiation, generate DC current, through Inverter, have the function of charging into the charged unit, converting DC/AC to serve the load in the home or grid.

Reconfiguration system is a device that increases the performance of the solar energy system in inhomogeneous radiation conditions, installed in front of the power adapter.

In this article, the author presents a restructuring strategy for the solar energy system based on TCT connection configuration, thereby proposing a general mathematical model for two optimal problems in the restructuring strategy. 


\section{Strategy for Restructuring TCT Connection Circuit}

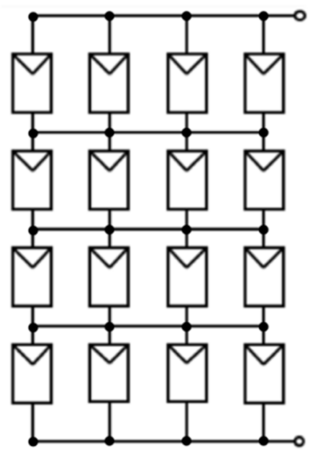

Figure 1. TCT connection circuit.

The strategy of restructuring the connection of Photovoltaic panels for TCT connection circuit was presented by the author in the research projects [11-14], summarizing the restructuring strategy as follows.

The TCT connection circuit consists of parallel connection profiles, parallel circuits connected in series and features Figure 1:

a. The maximum voltage of the parallel connection

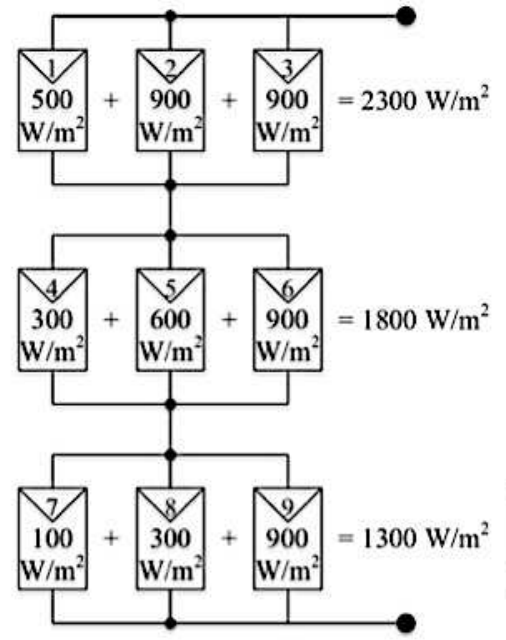

(a)

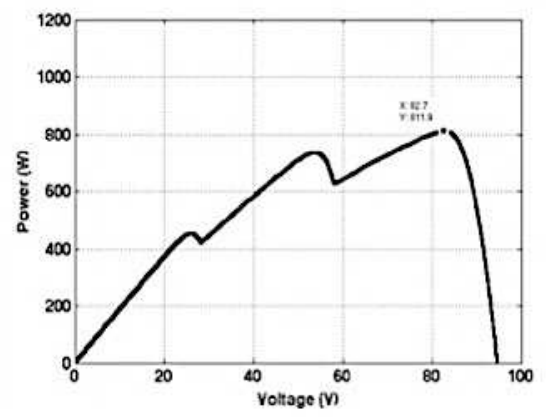

(c) circuits (in the TCT circuit) is not affected by the received lighting level of each photovoltaic panels.

b. The current generated by the series of parallel connected circuits will be proportional to the level of illumination received by each photovoltaic panel.

During the work, Photovoltaic panels are affected by partial coverage problems, possibly due to the shadow of buildings, cloud cover, snow, shadows of nearby objects, resulting in solar radiation received by each different photovoltaic panel. The method of radiation balance for TCT connection circuit is to rearrange the location of connection of photovoltaic panels in order to balance the total level of solar radiation at parallel connections in the TCT circuit as the example in Figure 3 [11]. In Figure 2, before the radiation balance, TCT circuits with total radiation at the rows are respectively $2300 \mathrm{~W} / \mathrm{m}^{2}, 1800 \mathrm{~W} / \mathrm{m}^{2}, 1300 \mathrm{~W} / \mathrm{m}^{2}$ (Figure $2 \mathrm{a}$ ). After changing the position of the photovoltaic panels as shown in the picture (photovoltaic panel 1 moves from row 1 to row 3), the total illumination level is $1800 \mathrm{~W} / \mathrm{m}^{2}$ at rows (Figure 2b). The maximum power before equilibrium is $811.9 \mathrm{~W}$ with misleading phenomenon (Figure 2c), after balancing, the system's maximum power increases to $1041 \mathrm{~W}$ ( $28.2 \%$ efficiency increase) with only one maximum point, avoid misleading phenomenon (Figure 2d).

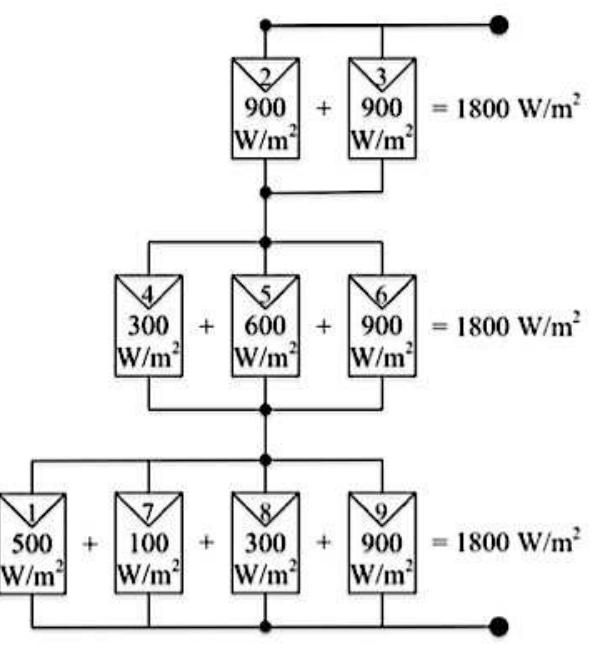

(b)

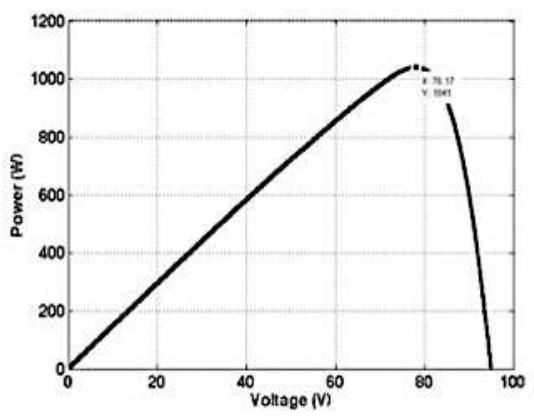

(d)

Figure 2. Example of radiation balance: (a) before balancing, (b) after balancing. Capacity chart: (c) before balancing with the misleading phenomenon, (d) after the balance is no longer misleading. 
The restructuring strategy, improving the working efficiency of the solar energy system may be generalized according to the flowchart at Figure 3.

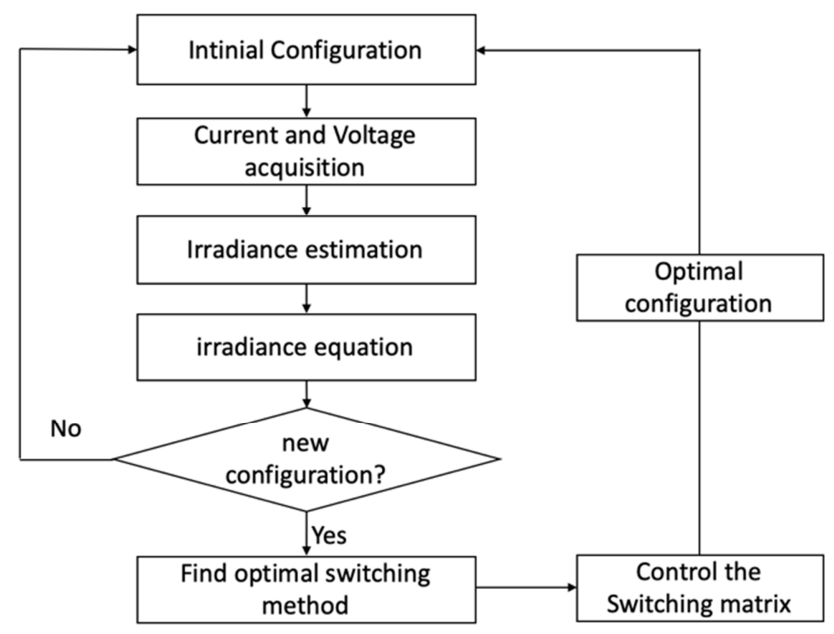

Figure 3. Flowchart of restructuring strategy.

The restructuring strategy includes the following steps:

Step 1: Measuring current and voltage for each photovoltaic panel.

Step 2: Based on currents, the estimated voltage of solar radiation received by each Photovoltaic panel.

Step 3: Applying the radiation balance algorithm, find the optimal connection configuration of the photovoltaic panels.

Step 4: Checking the new connection configuration compared to the original connection configuration. If it is the initial connection configuration, go back to step 1 . If it is a new connection configuration compared to the original configuration, go to step 5 .

Step 5: Applying algorithm to find optimal switching method. The optimal switching method is the one that uses the least number of locking and unlocking times, helping to extend the life of the switching matrix.

Step 6: Controlling switch matrix to lock and unlock according to the method searched in step 5, having the new connection configuration for the system performance that is best.

Thus, in the strategy of restructuring the connection of photovoltaic panels, including two main problems: Radiation balance problem and problem Select optimal switching method. In the following section, the author will present in detail and then propose a mathematical model for the above two optimal problems.

\section{Proposed Mathematical Model}

\subsection{Model of Mathematical Problem of Radiation Balance}

Considering the structure of the solar energy system of general TCT circuits including $m$ serial connection rows, each row is a circuit of parallel connected photovoltaic panels, the $i^{\text {th }}$ row consists of $n_{i}$, parallel connected photovoltaic panels as shown in Figure 4.

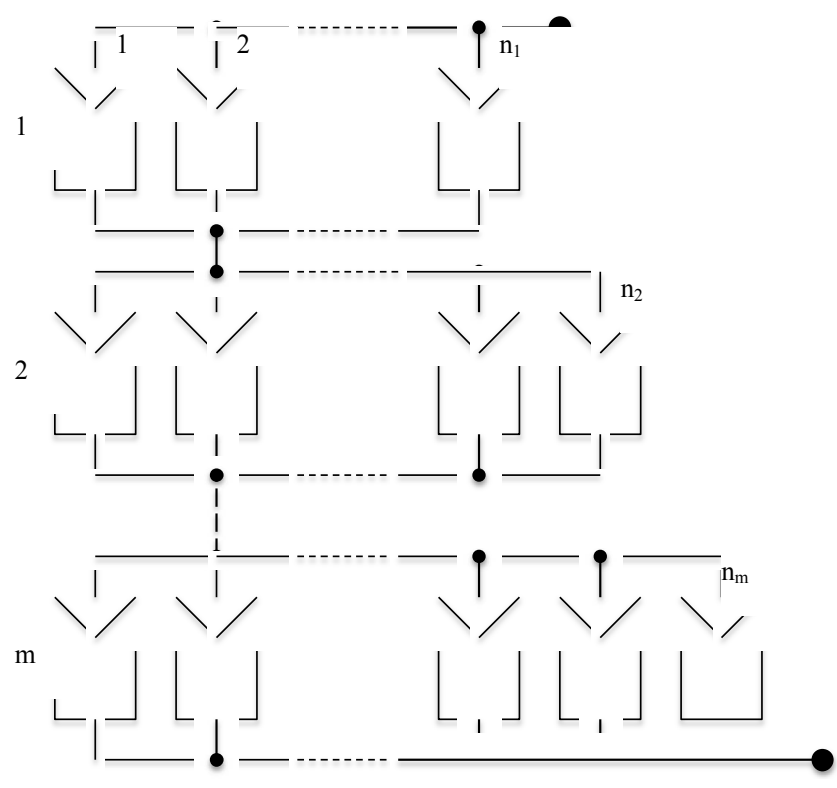

Figure 4. General TCT connection circuit.

If we consider $G_{i j}$ as radiation value of photovoltaic panel in row $i$ and column $j$ in TCT circuit. Total radiation level of row $i$ is $G i$ is determined by the formula:

$$
G_{i}=\sum_{j=1}^{n_{i}} G_{i j}
$$

Equilibrium index (EI) [15] for each connection configuration is calculated by the formula:

$$
E I=\max _{i=1, m}\left(G_{i}\right)-\min _{i=1, m}\left(G_{i}\right)
$$

Which configuration has the smallest EI index is the optimal configuration to be selected.

General objective function of the method:

$$
E I=\max _{i=1, m}\left(G_{i}\right)-\min _{i=1, m}\left(G_{i}\right) \rightarrow 0
$$

Binding conditions:

$$
\left\{\begin{array}{c}
n_{1}+n_{2}+n_{3}+\ldots+n_{m}=n \\
G_{i 1}+G_{i 2}+G_{i 3}+\ldots+G_{i n_{i}}=G_{i} \\
n_{i}>0 \\
G_{i j} \geq 0 \\
i=\overline{1, m} ; j=\overline{1, n_{i}}
\end{array}\right.
$$

Where:

EI: Equilibrium index

$n$ : Total number of photovoltaic panels

$m$ : Number of rows in TCT circuit

$n_{i:}$ Number of photovoltaic panels at $i^{\text {th }}$ row

$G_{i j:}$ Radiation value at photovoltaic panels in rows $i$ and $j$. 
$G_{i:}$ Total radiation at row $i$

Configuration satisfies the objective function of the method Radiated equilibrium is the configuration for the performance of the solar energy system is the largest. Calling $G$ the radiation matrix with the initial connection configuration, G_OP is the radiation matrix with the optimal connection configuration. In the next section, the author will present the problem of selecting the switching method from the initial configuration ( $G$ matrix) to the optimal configuration ( $G \_O P$ matrix).

\subsection{Mathematical Model Problem to Select the Optimal Switching Method}

During the study process, the author found that there were very few researches on the selection of the optimal switching method, in order to increase the life of the switching matrix, the problem of selecting the optimal switching configuration was working. Proposals proposed at, are described and constructed in the following section of the mathematical model [11].

\subsubsection{Switching Matrix Dynamic Electrical Scheme (DES)}

In fact, in the current solar energy systems, photovoltaic panels are fixedly connected, that is, the photovoltaic panels are physically connected to each other with the number of fixed or parallel fixed designs according to the design, in the process. The working cannot change the connection, ie, it does not change the connection position of photovoltaic panels in the connection circuit automatically. So that the photovoltaic panels to change the connection structure automatically, DES switching matrix is required.

DES switching matrix (Figure 6) has been proposed in [16] in order to change the connection customization of the solar energy system, from the initial connection configuration, through the locking and unlocking operations, there will be a new connection circuit with any structure.

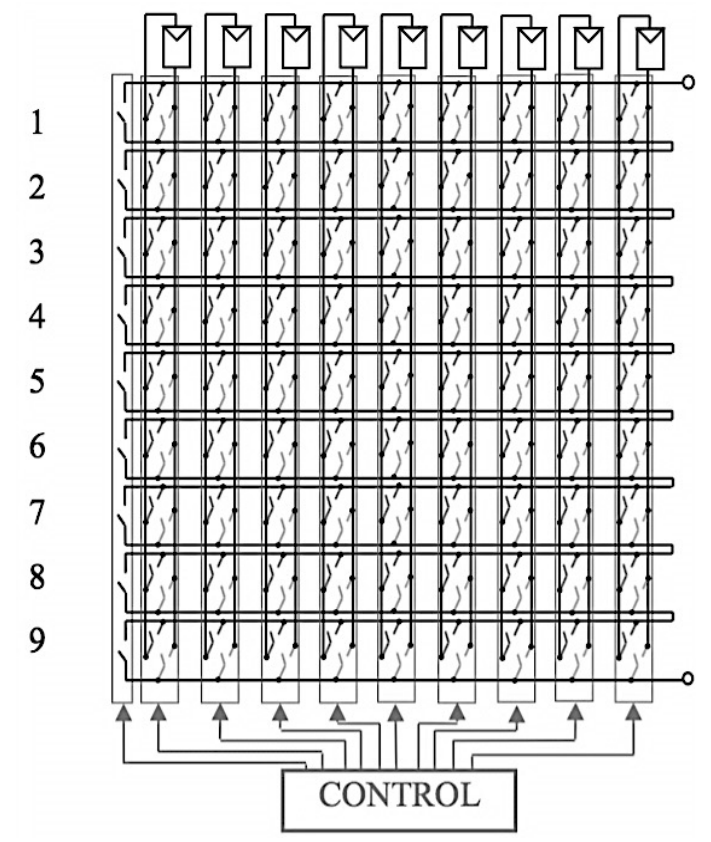

Figure 5. Dynamic Electrical Scheme (DES) Switching matrix [16].
Example of DES switching matrix in Figure 6:

a. To get the connection circuit as Figure 6a: Switching matrix Figure $6 \mathrm{~b}$ lock of photovoltaic panel No. 1 and No. 2 is closed in row 1, lock photovoltaic panel No. 3 and No. 4 close in row 2, other locks are opened.

b. To get connection circuit like Figure 6c: Switching matrix Figure 6d lock photovoltaic panel No. 1, No. 2 and No. 3 are closed in row 1 , lock photovoltaic panel No. 4 closed in row 2, other locks are opened.

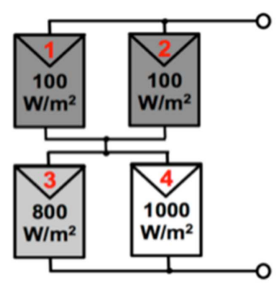

(a)

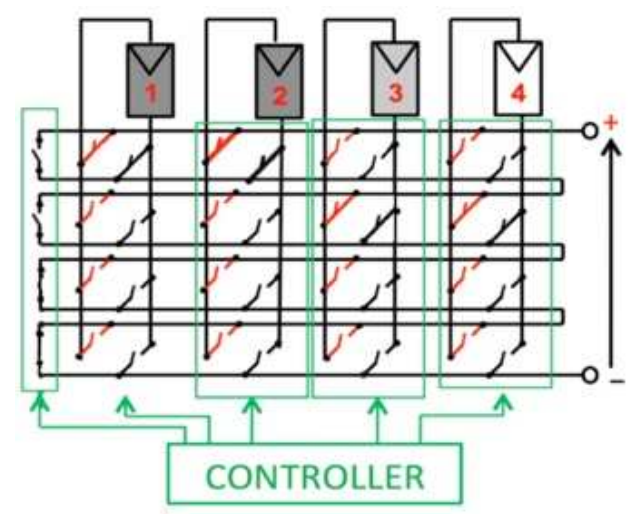

(b)

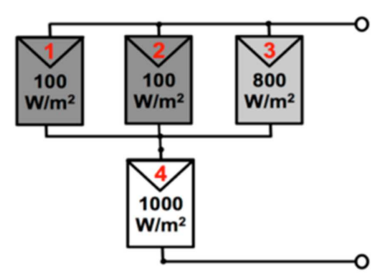

(c)

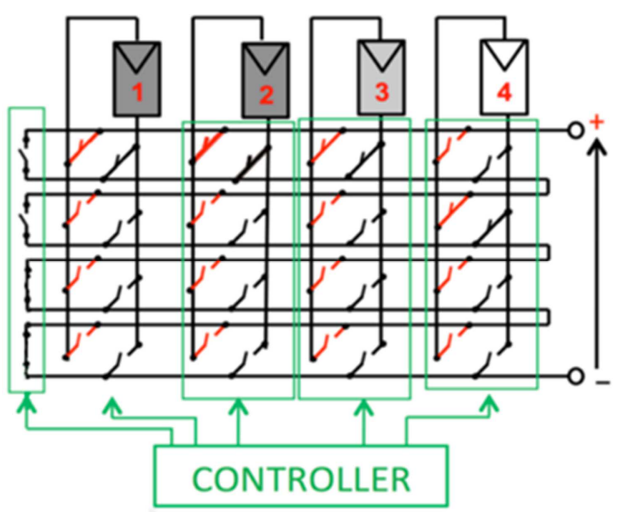

(d)

Figure 6. The Dynamic Electrical Scheme (b-d) switching matrix corresponds to the connection configuration (a-c). 
Thus, through the DES switching matrix, from any TCT connection circuit can initially change the connection to the general TCT circuit as Figure 4.

Consider the general DES switching matrix for $n$ photovoltaic panels as Figure 7.

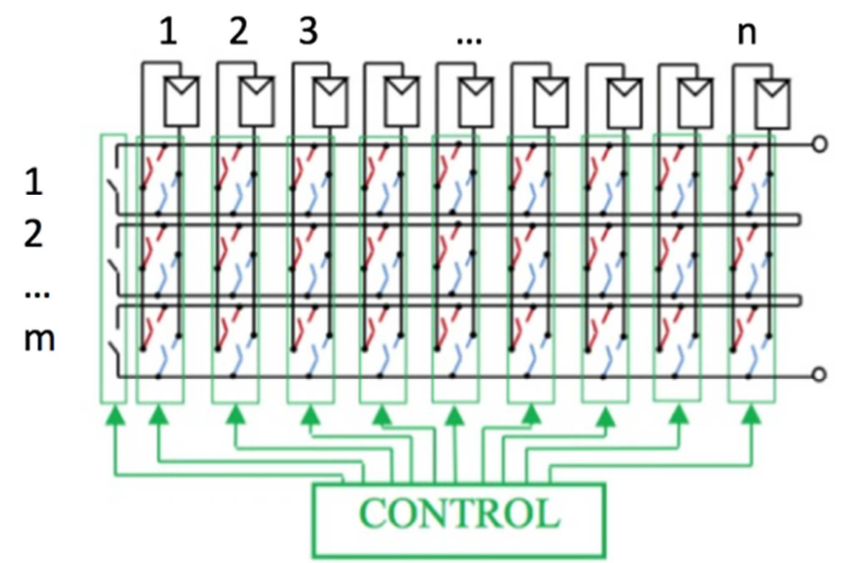

Figure 7. Matrix switch DES general for $n$ photovoltaic panels, $m$ serial circuits.

DES switching matrix is designed with circuit locking and unlocking, including 2 main parts:

a. The first part consists of the leftmost single key which acts to separate each row in the TCT circuit. Calling the array of $Q$ values with m elements corresponding to the number of locking and unlocking times of these single keys, the single key that opens and closes in every row with double-closed lock, the single left-open lock, all open-double lock then the left single lock is closed.

b. The second part consists of $m \times n$ double key located in the middle of the DES switching matrix, which acts to change the position of connecting photovoltaic panels in parallel circuits. Calling the value matrix $S$ consists of $m$ rows and $n$ columns corresponding to the number of locking and unlocking times of these double keys.

General number of unlocking times of switching matrix shown in. Table 1.

Table 1. $Q$ array and $S$ matrix represent the number of locking and unlocking of the switching matrix.

\begin{tabular}{llllll}
\hline \multirow{2}{*}{ Row index } & \multicolumn{2}{l}{ Column index } & & & \\
\cline { 2 - 6 } & & $\mathbf{1}$ & $\mathbf{2}$ & $\ldots$ & $\mathbf{n}$ \\
\hline 1 & $\mathrm{Q}_{1}$ & $\mathrm{~S}_{11}$ & $\mathrm{~S}_{12}$ & $\ldots$ & $\mathrm{S}_{\mathrm{nn}}$ \\
2 & $\mathrm{Q}_{2}$ & $\mathrm{~S}_{21}$ & $\mathrm{~S}_{22}$ & $\ldots$ & $\mathrm{S}_{2 \mathrm{n}}$ \\
$\ldots$ & $\ldots$ & $\ldots$ & $\ldots$ & $\ldots$ & $\ldots$ \\
$\mathrm{m}$ & $\mathrm{Q}_{\mathrm{m}}$ & $\mathrm{S}_{\mathrm{m} 1}$ & $\mathrm{~S}_{\mathrm{m} 2}$ & $\ldots$ & $\mathrm{S}_{\mathrm{mn}}$ \\
\hline
\end{tabular}

During the operation of the solar energy system and the restructure, after each restructuring, the number of locking and unlocking times of the switching matrix changes. In this study, the authors are interested in minimizing the number of locking and unlocking times of the double keys.

Convention on the number of times to close and unlock:

a. At the beginning:

$$
S_{i j}=0 \quad \forall i=\overline{1, m} ; j=\overline{1, n}
$$

b. During the operation, when changing the position of a photovoltaic panel $p$ from row $i$ to row $j$, number of locking and unlocking times of the $S$ matrix changes as follows:

$$
\begin{aligned}
& S_{i p}=S_{i p}+1 \\
& S_{j p}=S_{j p}+1
\end{aligned}
$$

Thus, calling the photovoltaic panel number to change position is $z$, the number of locking and unlocking times of switching matrix is $2 \mathrm{x} z$.

c. During operation, when detecting new connection configuration better than the old connection configuration, the system automatically changes the connection configuration.

\subsubsection{Problem of Optimal Switching Configuration Selection Method}

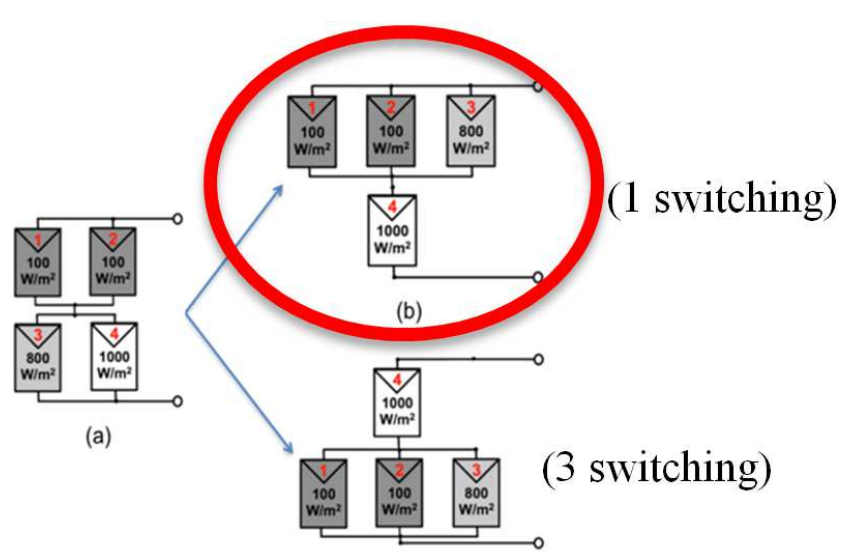

(c)

Figure 8. Example of radiation balance configuration but with different switching times.

The problem of selecting the optimal switching configuration is applied after the results of the radiation equilibrium problem, i.e the initial solar radiation matrix $G$ and the solar radiation matrix $G_{-} O P$ after radiation balance. The purpose is to control the switching matrix from the original configuration $G$ to the $G \_O P$ radiation equilibrium configuration so that the number of locking and unlocking times of the switching matrix $S$ is least.

In studies by Quesada [17], Romano [16] proposed radiation balance algorithms, but have not yet mentioned finding the optimal switching method that switching methods are used entirely based on the system. The results of the problem find the optimal connection structure, leading to a large number of switches, not interested in the lifespan of the switching matrix.

Table 2. Matrix $G$ (a) and matrix G_OP (b).

\begin{tabular}{lllll}
\hline 100 & 100 & 1000 & & \\
800 & 1000 & 100 & 100 & 800 \\
(a) & & (b) & & \\
\hline
\end{tabular}

Figure 8 is an example of the optimal switching 
configuration search method. Figue $8 \mathrm{a}$ is the initial connection structure of the system (matrix G corresponds to Table 2a). Figure $8 \mathrm{~b}$ and Figure $8 \mathrm{c}$ are two different structures of the same matrix $G_{-} O P$ (Table $2 \mathrm{~b}$ ) - the result of the problem of finding the radiation balance structure.

It is easy to realize that switching from the initial structure a to the radiation balance structure $b$ takes only 1 time of relocating photovoltaic panel (transferring the photovoltaic panel 3 from row 2 to row 1), number of lock locking and unlocking times is 2 .

On the other hand, from the original structure to the radiation balance structure Figure $8 \mathrm{c}$, it takes 3 times to change the position of the locked photovoltaic panel (transfer the photovoltaic panels 1, 2 from row 1 to row 2; transfer pin 4 from row 2 to row 1 ), in this case, the number of locking and unlocking times is equal to 6 .

Notice that the number of unlocking times depends on the order of the rows in the $G_{-} O P$ matrix, so which $G_{-} O P$ configuration with the least number of locking and unlocking times will be selected.

Calling $M I_{\text {stepk }}$ is the number of unlocking times of the $k^{\text {th }}$ restructuring, $(S i j)$ step $k$ is the total number of unlocking sessions of the key with the index $i$ and column $j$ in the switching matrix, up to the end of the reconstruction. kth structure. $M I_{\text {stepk }}$ is calculated according to the formula:

$$
M I_{\text {stepk }}=\sum_{\substack{i=1 \\ j=1}}^{\substack{i=m \\ j=n}}\left(S_{i j}\right)_{\text {stepk }}-\sum_{\substack{i=1 \\ j=1}}^{\substack{i=m \\ j=n}}\left(S_{i j}\right)_{\text {stepk-1 }}
$$

Where:

$m$ : Number of rows in TCT circuit

$n$ : Number of photovoltaic panels

$M I_{\text {stepk: }}$ Number of unlocking times for the $k^{\text {th }}$ restructuring.

During operation, maybe the photovoltaic panels are often most covered that will be switched most, leading to an imbalance in the number of full-matrix switches, at this time the matrix life will depend on the switch lock the most. So the problem arises: In many cases, the configuration with the number of locking and unlocking times is not necessarily selected, the other connection configuration must be selected, so as to minimize the number of locking and unlocking times of the lock with the highest switching frequency.

$\left(M I_{\text {min }}\right)_{\text {stepk }}$ is the minimum number of locking and unlocking times of the $k^{\text {th }}$ restructuring. We have the objective function of the problem of selecting the optimal switching method:

$$
\left\{\begin{array}{c}
\max _{\substack{i=1, m \\
j=1, n}}\left(S_{i j}\right)_{\text {stepk }}-\max _{\substack{i=1, m \\
j=1, n}}\left(S_{i j}\right)_{\text {stepk-1 }} \rightarrow 0 \\
M I_{\text {stepk }}=\sum_{\substack{i=m \\
j=m}}\left(S_{i j}\right)_{\text {stepk }}-\sum_{\substack{i=1 \\
j=1}}^{j=n}\left(S_{i j}\right)_{\text {stepk-1 }} \rightarrow\left(M I_{\text {min }}\right)_{\text {stepk }}
\end{array}\right.
$$

Bind to:

$$
\left\{\begin{array}{c}
S_{i j} \geq 0 \\
\sum_{\substack{i=m \\
j=n \\
j=1}}^{j=n}\left(S_{i j}\right)_{\operatorname{step~} 0}=0
\end{array}\right.
$$

Where:

$\left(M I_{\text {min }}\right)_{\text {stepk }}$ : The number of unlocking times is the least for the $k^{\text {th }}$ restructuring.

After selecting the connection configuration and the optimal switching method according to the two problems that have built the mathematical model above, the controller is designed to control the keys in the closed switch matrix properly, switch from the initial connection configuration to the optimal connection configuration.

\section{Conclusion}

In this article, the author focuses on analyzing and proposing mathematical models for two main problems in previous studies, developing objective functions and clear constraints, which are the rationale for evaluation of the quality and accuracy of the proposed algorithms, thereby developing more optimal algorithms than previous algorithms. Next step of the research, the authors will focus on the configuration of SP type and the problem of reconfiguration models for large solar power farms.

\section{Acknowledgements}

The authors wish to thank the Institute of Energy Science (IES) and Vietnam Academy of Science and Technology (VAST) for their support to the research activity of Project "Research on completing the technology, constructing and transferring the model of exploiting and proper using solar and wind energy for production and daily living in Central Highland", TN17/C03.

\section{References}

[1] Faiza Belhachat, Cherif Larbes, Global maximum power point tracking based on ANFIS approach for PV array configurations under partial shading conditions. Renewable and Sustainable Energy Reviews, 2017. 77.

[2] Boualem Bendib, Hocine Belmili, Fateh Krimb, A survey of the most used MPPT methods: Conventional and advanced algorithms applied for photovoltaic systems. Renewable and Sustainable Energy Reviews, 5/2015. 45.

[3] Mohamed A. Eltawil, Zhengming Zhao, MPPT techniques for photovoltaic applications. Renewable and Sustainable Energy Reviews, 12/2013. 25: p. 793-813.

[4] Balaji Veerasamy, Wataru Kitagawa, Takaharu Takeshita, MPPT method for PV modules using current control-based partial shading detection, in 2014 International Conference on Renewable Energy Research and Application (ICRERA). 2014. 
[5] Merwan Saadsaoud, Abbassi Hadj AHmed, Kermiche Salah, Study of Partial Shading Effects on Photovoltaic Arrays with Comprehensive Simulator for Global MPPT control. International Journal of Renewable Energy Research-IJRER, 2016. 6 (2)

[6] Subhashree Choudhury, Pravat Kumar Rout, Adaptive Fuzzy Logic based MPPT Control for PV System Under Partial Shading Condition. International Journal of Renewable Energy Research-IJRER, 2015. 5 (4).

[7] Subhash Chander, A. Purohit, Anshu Sharma, S. P. Nehra, M. S. Dhakaa, Impact of temperature on performance of series and parallel connected mono-crystalline silicon solar cells. Energy Reports, 2015. 1: p. 175-180.

[8] F. Reis, C. Guerreiro, F. Batista, T. Pimentel, M. Pravettoni, J. Wemans, G. Sorasio, M. C. Brito, Modeling the Effects of Inhomogeneous Irradiation and Temperature Profile on CPV Solar Cell Behavior. IEEE Journal of Photovoltaics, 2015. 5 (1): p. 112-122.

[9] Joseph J. Wysocki, Paul Rappaport, Effect of Temperature on Photovoltaic Solar Energy Conversion. Journal of Applied Physics, 2004. 31 (571).

[10] Priyanka Singh, S. N. Singh, M. Lal, M. Husain, Temperature dependence of $I-V$ characteristics and performance parameters of silicon solar cell. Solar Energy Materials and Solar Cells, 2008. 92 (12): p. 1611-1616.

[11] Eleonora Riva Sanseverino, Ngo Ngoc Thanh, Marzia Cardinale, Vincenzo Li Vigni, Domenico Musso, Pietro Romano, Fabio Viola, Dynamic programming and Munkres algorithm for Optimal Photovoltaic Arrays Reconfiguration. Solar Energy, 12/2015. 122: p. Pages 347-358.
[12] Ngo Ngoc Thanh, Nguyen Phung Quang, Pham Thuong Cat, Improved control algorithm for increase efficiency of photovoltaic system under non-homogeneous solar irradiance. Special issue control and automation, 2016. 16: p. 12.

[13] Thanh Ngo Ngoc, Quang Nguyen Phung, Linh Nguyen Tung, Eleonora Riva Sanseverino, Pietro Romano, Fabio Viola, Increasing efficiency of photovoltaic systems under nonhomogeneous solar irradiation using improved Dynamic Programming methods. Solar Energy, 2017. 150: p. 325-334.

[14] Ngo Ngoc Thanh, Nguyen Phung Quang, Simulation of reconfiguration system using Matlab-Simulink environment. Journal of Computer Science and Cybernetics, 2018. Accepted.

[15] Damiano La Manna, Vincenzo Li Vigni, Eleonora Riva Sanseverino, Vincenzo Di Dio, Pietro Romano, Reconfigurable electrical interconnection strategies for photovoltaic arrays: A review. Renewable and Sustainable Energy Reviews, 2014.

[16] P. Romano, R. Candela, M. Cardinale, V. Li Vigni, D. Musso, E. Riva Sanseverino, Optimization of photovoltaic energy production through an efficient switching matrix. Journal of Sustainable Development of Energy, Water and Environment Systems, 2013. 1 (3): p. 227-236.

[17] Guillermo Velasco, Francesc Guinjoan, Robert Pique, Electrical PV Array Reconfiguration Strategy for Energy Extraction Improvement in Grid-Connected PV Systems. IEEE Transactions on Industrial Electronics, 2009. 56 (11): p. 43194331. 